\title{
Comment on: Broaching goals-of-care conversations in advancing pediatric cancer
}

\author{
Chirag Shah ${ }^{1}$, Kayden Chahal ${ }^{1}$, Ashni Chani $^{1}$, and Tejas Kotwal ${ }^{1}$ \\ ${ }^{1}$ King's College London
}

September 25, 2021

Comment on: Broaching goals-of-care conversations in advancing pediatric cancer

Chirag Shah[1], Kayden Chahal[1], Ashni Chani[1], Tejas Kotwal[1]

[1] Department of Life Sciences and Medicine, King's College London, England, SE1 9RT

Word Count: 464 words

Corresponding Author: Chirag Shah

Mobile Number: (+44)07446146162

E-mail Address: chirag.shah@kcl.ac.uk

Dear Editor,

We read with great interest the article by Kaye et al. that highlights an incredibly important and sensitive area of pediatric care planning ${ }^{1}$. As final year medical students from the UK who have completed a twomonth pediatric placement, we were fortunate to have the opportunity to observe similar discussions and would like to share our own experiences in relation to this insightful article.

It is critical to have goals of care conversations with pediatric cancer patients and their families to ensure they receive personalised support. These discussions have been linked with a higher quality of care, a decrease in undesirable aggressive treatment and better bereavement adjustment for caregivers ${ }^{2}{ }^{3}$. From our own experiences in sitting in on these conversations, we appreciate the difficulty in navigating through these often highly emotive discussions. It should be noted that there should not be a "one gloves fits all" approach as each conversation must be tailored towards each individual patient. We commend the authors for identifying several strategies to help clinicians broach these conversations.

The study cohort demographics shows that $47 \%$ of the patients were under the age of 12 . During our placement we found it was difficult to engage children of this age for these types of conversations and we often solely relied upon parents' views. It is essential to ascertain what patients and their families expect from these discussions to prevent missed opportunities from occurring. These can include inadequately responding to concerns over disease progression or addressing concerns by focusing on optimistic talk of future ${ }^{4}$.

The paper highlights that goals of care conversations occur in the setting of advancing pediatric cancer care, however it is unclear when in the course of the illness these discussions start and how frequent they are. It is imperative that these discussions are initiated early, so that clinicians can align the care provided with what is most important for the patient. With the dynamic nature of cancer, it is vital that these conversations occur regularly, to allow the patient and their family to express any concerns when there is a change to their condition. It has been outlined that there are sometimes barriers that are perceived by clinicians that hinder 
discussions being initiated that need to be addressed. These can include the patients lack of capacity to make decisions, patients and family members' difficulty accepting a poor prognosis and understanding the complications and limitations of life-sustaining treatment ${ }^{5}$.

Consequently, further research is needed to explore the frequency of these conversations, barriers that prevent conversations being initiated and to determine what patients and their families expect, to prevent missed opportunities from occurring. In summary, it is vital that this area of pediatric cancer care continues to be researched. We hope our comments are useful for any future studies that take place.

\section{References}

1. Kaye EC, Woods C, Velrajan S, Lemmon ME, Baker JN, Mack JW. Broaching goals-of-care conversations in advancing pediatric cancer. Pediatr Blood Cancer . 2021;68(10):e29270. doi:10.1002/PBC.29270

2. AA W, B Z, A R, et al. Associations between end-of-life discussions, patient mental health, medical care near death, and caregiver bereavement adjustment. JAMA . 2008;300(14):1665-1673. doi:10.1001/JAMA.300.14.1665

3. Bansal A, Monk A, Norman M, Fingas S. Improving medical students' confidence in end-of-life consultations. Clin Teach . 2020;17(6):705-710. doi:10.1111/TCT.13214

4. Knutzen KE, Sacks OA, Brody-Bizar OC, et al. Actual and Missed Opportunities for End-of-Life Care Discussions With Oncology Patients: A Qualitative Study. JAMA Netw Open . 2021;4(6):e2113193e2113193. doi:10.1001/JAMANETWORKOPEN.2021.13193

5. You JJ, Downar J, Fowler RA, et al. Barriers to Goals of Care Discussions With Seriously Ill Hospitalized Patients and Their Families: A Multicenter Survey of Clinicians. JAMA Intern Med . 2015;175(4):549-556. doi:10.1001/JAMAINTERNMED.2014.7732 